\title{
Hacia una estructuración teórica de la Psicología Diferencial
}

\section{José A. Forteza Méndez, y José M. Prieto Zamora}

Universidad Complutense de Madrid

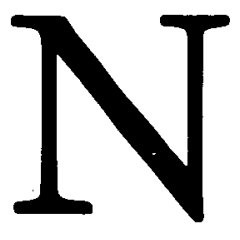

No deja de llamar la atención el hecho de que últimamente se ha observado un cierto desdén hacia los enfoques diferenciales entre los psicólogos-académicos, al ocuparse de la crisis del paradigma y de los modelos ejemplares de la Psicología de la Conducta entendida como quehacer científico. Y quizá el motivo radique en que, durante los pasados cien años de psicología, el paradigma R-R se ha situado al margen de las polémicas de escuela y, por ende, no ha sufrido ninguna «revolución científica» al estilo de las señaladas por T. S. Kuhn (1962) y reseñadas recientemente por A. Caparrós (1980) en el ámbito psicológico. Este reconoce que «el paradigma R-R no ha conocido en su historia auténticas crisis paradigmáticas» y que las polémicas «son intra y no interparadigmáticas» a la hora de interpretar resultados e investigaciones.

Una de las razones de este hecho radica, sin duda, en que el objeto de la psicología diferencial no ha sido nunca la conducta en abstracto, sino siempre abordada en el contexto de un marco aplicado y concreto, lo más próxima posible a sus manifestaciones en la vida real y rehuyendo, tajantemente, los estrechos márgenes de una ciencia ceñida a experimentos de laboratorio. Por ello, quienes con más frecuencia recurren a la psicología diferencial con vistas a recabar de ella un mapa de. conocimientos sistemáticos, coherentes y operativos son, por contra, los psicólogosprofesionales. Estos tienen que enfrentarse al tema de la conducta bumana en el contexto de problemas y circunstancias que deben ser resueltas y afrontadas funcional y eficazmente. Esto ha traído como consecuencia que la psicología diferencial esté sesgada, en su discurso, hacia logros y constataciones empíricas, brillando por su ausencia una urdimbre teórica cabal y comprensiva. 


\section{Estudios}

Ciertamente la psicología diferencial, desde hace algunos años, ha adoptado como peculiares los métodos de análisis estadístico multivariado toda vez que permiten estudiar cuasi-experimentalmente una situación dada con un mínimo de alteraciones. Con una sola recogida de datos, en función de un correcto muestreo de sujetos y de variables, se consigue:

1. Una optimación del número de relaciones observadas y tomadas como objeto de estudio.

2. Una identificación cuantitativa (las saturaciones) y cualitativa (los factores) de los distintos focos de influencia y determinación que subyacen a la varianza obtenida.

3. Una ponderación de la importancia de las distintas dimensiones identificadas bien mediante una «rotación referida a un criterio», bien merced a «la estructura simple», bien a través de una contrastación multirrasgo-multimiétodo, bien comparando las variables relevantes con las que se arremolinan en torno al hiperplano, etc...

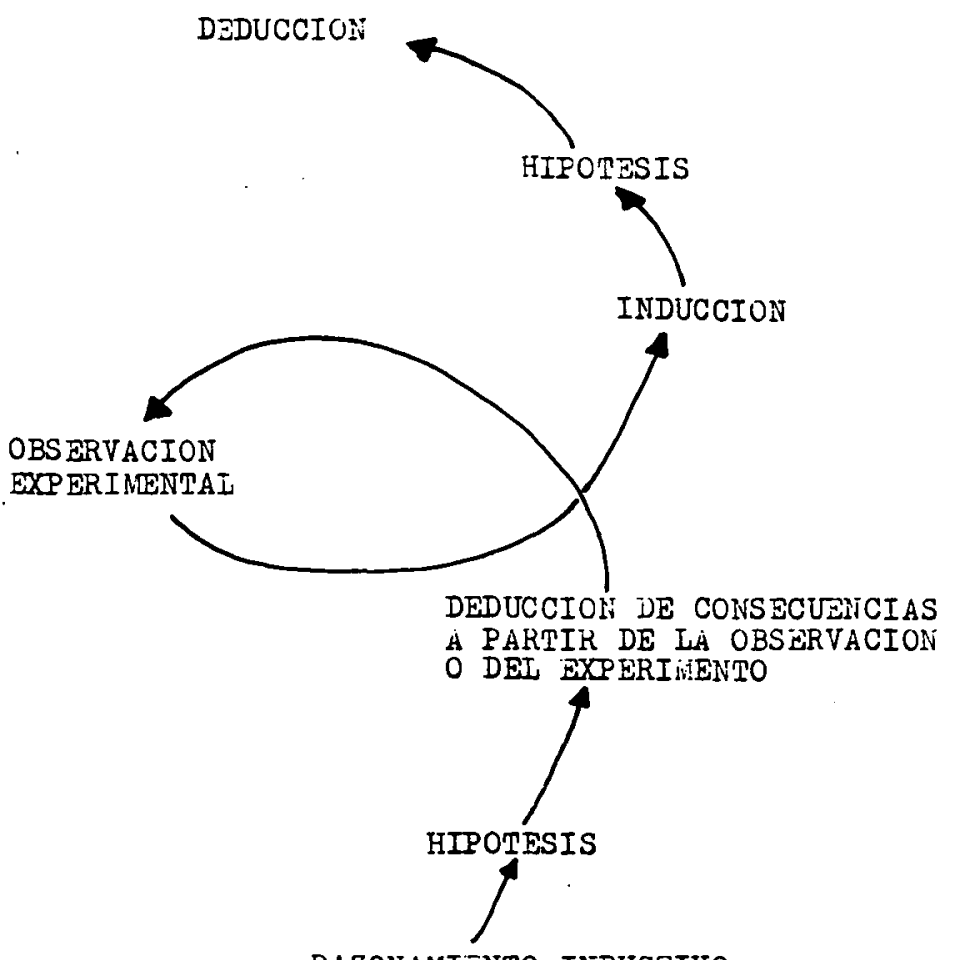

RAZONAWIENTO INDUCTIVO

DE CIERTAS 'REGULARIDADES'

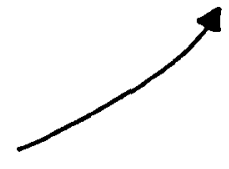


4. Una verificación de los constructos hipotéticos identificados sometiéndolos a prueba -en el laboratorio o en la vida real - utilizando gamas de variables representativas siguiendo sea esquemas manipulativos-bivariados, sea la regresión múltiple respecto a criterio (según los pesos betta de la ecuación de especificación), sea, en fin, las oscilaciones globales a dividir las muestras en función de la edad, el sexo, la profesión, el rendimiento, la creatividad, la problemática clínica y/o escolar, etc...

El modelo científico que subyace a esta metodología diferencial es no, pues, el mero Hipotético-Deductivo clásico, sino más bien una modificación del mismo al que acertadamente R. B. Cattell (1966) ha denominado «la espiral Inductivo-Hipotético-Deductiva» y que queda debidamente ilustrada en la figura 1.

El análisis multivariado, especialmente factorial, resulta particularmente adecuado a la hora de desglosar inductivamente la maraña de contenidos y de datos que suministra la realidad, sobre todo en el primer escalón de esta espiral, puesto que permite la identificación de los constructos empíricos desconocidos - los factores- basándose exclusivamente en la varianza común recopilada.

Joseph R. Royce y su equipo de colaboradores sitúan precisamente en este nivel el análisis factorial a la hora de construir la armazón de su teoría multifactorial-sistemática. Si bien es preciso reconocer ciertas complicaciones de tipo preferentemente matemático al fijar el número de factores significativos así como al decidir el tipo de algoritmo adecuado para una rotación determinada, el análisis factorial consigue identificar los sucesivos «determinantes de la covariación» que se muestran invariantes» y arepetibles a pesar de las variaciones», pudiendo «servir como variables dependientes o independientes» (Royce, 1963). Así se da pie a su clarificación como construtos teóricos con relevancia y contenido psicológico coherente. La figura 2 ilustra lo que se está explicitando, de un modo claro y logístico.

FIGURA 2: Esquema heuristico en la investigaciön Multifactorial (K. Pawlik 1973).

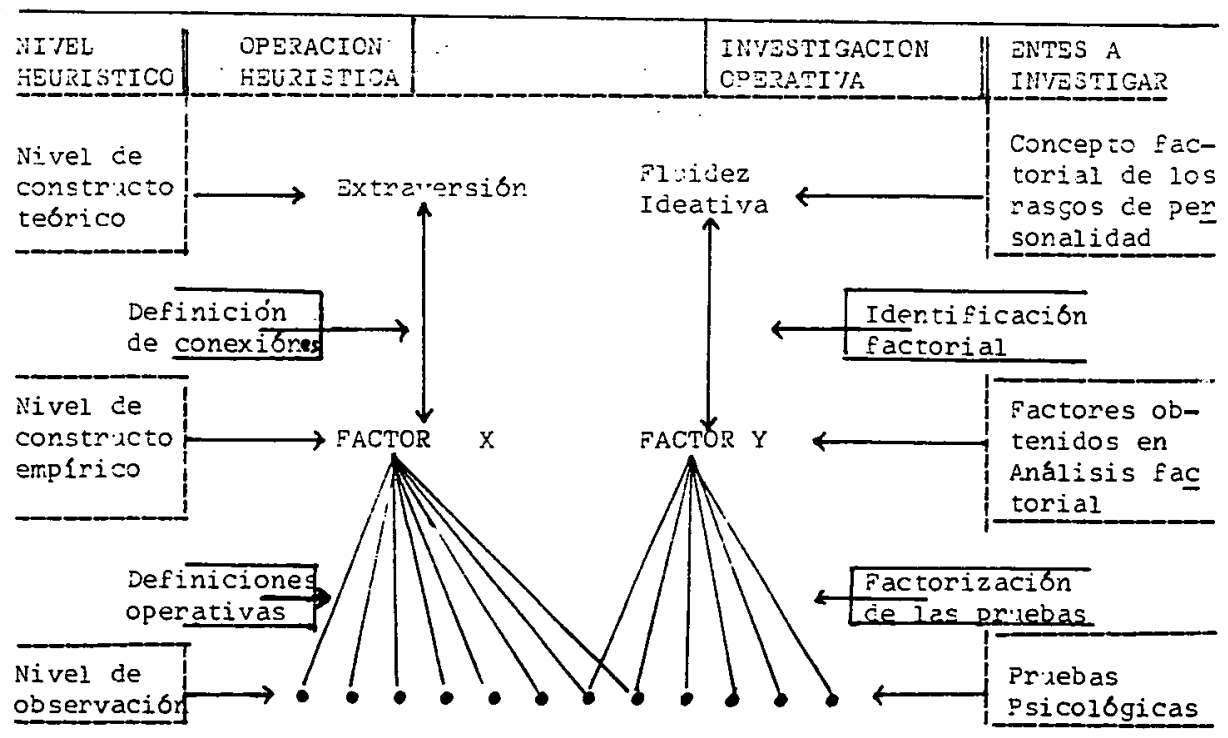




\section{Estudios}

Los factores que se extraen del análisis matemático-estadístico de la matriz de datos son meros factores $\mathrm{X}$ o $\mathrm{Y}$ que son asumidos, en la investigación, como simples constructos empíricos. Estos constructos deberán reproducirse utilizando muestras diversas de sujetos así como muestras distintas de variables (entre las que se mantendrán las más relevantes como claves de identidad en los sucesivos trabajos); por otra parte, el psicólogo, mediante sus conocimientos teóricos en el área cocernida les aplica un epígrafe provisional basándose en el contenido de las variables que saturan idóneamente en torno al eje factorial subyacente, verificando tanto sus conexiones empíricas como las expresamente correlacionadas.

Royce y sus colaboradores han sido particularmente exigentes a la hora de aceptar los factores que debían incorporarse a cada uno de los subsistemas. El criterio seguido ha sido el de un «alto nivel de invarianza», comprobada mediante alguno de los siguientes requisitos (Royce, 1973):

1. Que el factor haya sido identificado en dos o más investigaciones (en dos o más laboratorios o institutos independientes).

2. Que se haya conseguido aplicarlos a través de programas masivos e intensos de investigación.

3. Que se haya llevado a cabo una cierta manipulación experimental consistente en el constructo teórico previamente identificado.

Estas condiciones son lo suficientemente restrictivas como para salir al paso de la profusa proliferación de factores que han emergido incondicionalmente en la esfera temperamental-motivacional, así como en la cognitivo-aptitudinal. Se logra así una cierta taxonomía estricta y, a la par, rica en contenidos descriptivo-explicativos de la conducta humana. Por ende, resulta particularmente útil de cara a la psicología aplicada por la inmediatez de convertir las variables utilizadas en instrumentos de medición.

Ahora bien, las clasificaciones taxonómicas, particularmente en lo que concierne a los rasgos aptitudinales, temperamentales y dinámicos han sido criticadas abundantemente como excesivamente estáticas y rígidas. Ciertamente en sus primeros trabajos los pioneros pusieron excesivo énfasis en el matiz de que se trataba de «factores comunes» y, por ello, extensibles a todo tipo de personas así como persistentes a lo largo de la vida del sujeto. Cattell, por ejemplo, tras ocuparse de la «esfera de la personalidad» y del «entramado dinámico» inició una serie de trabajos en torno a los estados de ánimo y, consecuentemente, los cambios que se operan en las personas. Tales esfuerzos le llevaron a distinguir factores que eran «tasgos» y factores que eran «rasgos del cambio», discerniendo sus respectivos patrones tanto cuantitativos como cualitativos. Esto le ha conducido a las siguientes matizaciones:

«Es preciso reconocer, no obstante, la existencia de tres conceptos: rasgos, estados y rasgos del cambio. Los rasgos del cambio forman parte del propio crecimiento o son simplemente fluctuaciones. Los rasgos pueden desarrollarse bien de un modo uniforme bien por etapas. La fluctuación o el desarrollo uniforme harán que el patrón de los rasgos del cambio se mimetice íntimamente con el patrón de los rasgos» (1).

Ahora bien, ciertamente el modelo multifactorial, aun cuando se ha ocupado del cambio en cuanto realidad psicológica, no ha logrado un encaje coherente del mismo, especialmente en lo que concierne a su aplicabilidad. De ahí que al centrarse en los procesos psicológicos los teóricos se hayan remontado de un lado hacia la 
Teoría General de Sistemas y de otro hacia la Teoría de los Procesos de Información. En J. P. Guilford ya estaba implícita esta tendencia a desarrollar su esquema respecto a la.Naturaleza de la Inteligencia. Lo mismo acaece en R. B. Cattell (1971), en una obra muy poco conocida en el mundo de habla hispana, cuando expuso su «teoría triádica del desarrollo de las aptitudes y su conexión con la teoría estructural del aprendizaje», estableciendo la distinción entre «capacidades generales» (g), «peculiaridades organizativas» (p), y «agentes» (a). Estas implicaciones se hacen aún más evidentes y tangibles en su último libro (Cattell, 1980), donde desarrolla «una teoría sistemática de la maduración y del aprendizaje estructurado», en el contexto de sus dos textos más recientes en los que establece un puente entre la teoría de la personalidad y la del aprendizaje estructural.

Royce y Powell, en Canadá, también han desembocado en este tipo de tratamiento del tema; en él vienen trabajando desde hace diez años en el Centro de Estudios Avanzados en Psicología Teórica de la Universidad de Alberta. Próximamente publicarán un libro donde se recogerán y glosarán las aportaciones de los numerosos colaboradores que han intervenido (gracias al apoyo financiero del «Comité Canadiense de Dotaciones»). El trabajo que se recoge en esta sección de Dossier, es una exposición sucinta del texto mencionado, viniendo a ser la recopilación de buena parte de las publicaciones y monografías en curso o ya difundidas; en él se presenta el cogollo de este nuevo enfoque de la Psicología Diferencial en términos Multifactoriales y Sistemáticos, recurriendo para su expresión a esquemas de procesos y flujos recabados de la Teoría de la Información.

La Teoría General de Sistemas entronca con los trabajos de L. von Bertalanffy $(1055,1962)$ en torno a la biología de los organismos, toda vez que se puede comprobar en los mismos que sus estructuras están orientadas de un modo funcional. La naturaleza ha consagrado sistemas reiterativos que permiten a los seres vivos alcanzar sus fines siguiendo ciertas pautas y estrategias que organizan y estructuran las peculiaridades de todo individuo en su interacción con el ambiente. Por su parte la Teoría de los Procesos de Información se adscribe a la orientación funcionalista del neoconductismo (Lindsay y Norman, 1972; Carrol y Payne, 1976; Scandura y Brainerd, 1978) que al abordar los procesos mentales adopta esquemas y referencias que se han mostrado operativos y viables en el ámbito de la programación y la informática.

En la conjunción de estos tres enfoques se consigue un marco explicativo en el que los factores permiten la identificación de las dimensiones más consistentes; los sistemas reagrupan coherentemente estas dimensiones según esferas de incidencia y de evaluación comportamental; finalmente los esquemas de procesos y flujos logran expresar funcionalmente el modo en que el individuo capta la información, la traduce a sus propios códigos, la transforma y la integra en sucesivas actuaciones y comportamientos. Brinda, pues, una urdimbre descriptivo-explicativa que permite esbozar una nueva «Psicología de la Individualidad» partiendo de lo que hay de común en las diferencias entre las personas. Esta Teoría Multifactorial-Sistemática ofrece el sustrato adecuado para llevar adelante una Psicología Aplicada centrada en lo que se conoce respecto al ensamblaje sistemático de las peculiaridades y características individuales siempre en interacción con el medio. De igual manera se consigue una reinterpretación sistemática de los muy numerosos trabajos en torno a la influencia de la herencia y el ambiente en la configuración de las diferencias interindividuales. Es éste uno de los apartados más concisos, elaborados y a la vez clarividentes por cuanto recupera datos provenientes de la investigación «naturaleza-en- 


\section{Estudios}

torno» de un lado, de las dotaciones psicofisiológicas inherentes a las peculiaridades organizativas de la sensomotricidad de otro, así como de las derivaciones psico-sociales de los estilos y valores. Todo ello abordado con discreción y sosiego, situándose al margen de polémicas más o menos emocionales, y conectando los hallazgos y constataciones con la teoría estructurada del aprendizaje.

Resulta a todas luces original que, en esta re-estructuración teórica de la Psicología Diferencial, se recupere el concepto de tipo, que parecía relegado al baúl de los recuerdos. El tipo, en este contexto, asume tanto las variaciones inter-individuales de tipo multidimensional (más bien estáticas) en cuanto a las similitudes entre grupos como las diferencias individuales en la secuencialidad de los procesos de información (más bien dinámicos). De esta suerte el concepto de personalidad-tipo, en cuanto patrón del supra-sistema psicológico total, aglomera y absorbe tanto el perfil de rasgos como la configuración temporal de estrategias y predisposiciones comportamentales que permiten el afrontamiento del entorno y sus exigencias.

De igual manera llama la atención el hecho de que, al abordar el subsistema de los estilos, se enfaticen tanto sus derivaciones cognitivas como afectivas. Esto implica que los estilos son entendidos como componentes de orden superior que inciden efectiva y cognitivamente en la integración de los fenómenos de la vida real. Intervienen, pues, en las situaciones diarias a manera de variables moderadoras que se activan discrecionalmente para dar cauce al ajuste.

En esta tesitura la Teoría Multifactorial-Sistemática recupera el concepto de personalidad en su sentido lato (véase al respecto Estudios de Psicología n ${ }^{\circ} 3$, Prieto, 1980), posibilitando una evaluación comprehensiva de la persona en función de los perfiles en cada uno de los subsistemas. Igualmente propicia un análisis de las situaciones siguiendo pautas socio-sistemáticas de flujo, con vistas a determinar diferencialmente en qué medida puede darse el ajuste y la adaptación comportamental de la persona en su medio.

Un aspecto susceptible de discusiones sin fin en esta teoría radica en que se haya recurrido a un modelo jerárquico en el modo de estructurar tanto el supra-sistema total (la personalidad) como cada uno de los sub-sistemas. Aparte del jerárquico pueden ser viables los modelos reticulares (propiciados en parte por Cattell y colaboradores, aunque a efectos de exposición opten también por los jerárquicos) o el de facetas propuesto por Guttman (1955), y adoptado por Guilford al pergeñar su estructura del intelecto.

Evidentemente el hecho de que los factores se ramifiquen jerárquicamente a partir de las rotaciones con estructuras oblicuas no deja de ser un artificio del análisis factorial mismo. De igual manera la cooperación entre factores al incidir en un rendimiento dado, secunda la viabilidad de los modelos reticulares. Desde el punto de vista heurístico el modelo de facetas permite agotar, sistemáticamente, todas las posibilidades de interacción entre cada una de las dimensiones involucradas en cada sub-sistema, pero como consecuencia el modelo se complejiza excesivamente desembocando en alternativas y aspectos que son más bien artificiales que naturales en el desenvolvimiento humano (como apuntan buena parte de los críticos de los trabajos de Guilford). El mismo Royce (1973) justifica su opción por el modelo jerárquico por cuanto se ciñe más a la metodología multifactorial utilizada y porque se muestra un esquema abierto a la incorporación de nuevos hallazgos y constataciones. 
«Mi enfoque no lleva implícita, a priori, la adopción de un orden jerárquico. Mi punto de vista personal en este asunto viene a ser que todos los modelos disponibles, incluido el mío, son casos especiales de un segmento particular de variables. Así cualquier tipo de jerarquía, estrato o cadena constituye un subconjunto en el seno de una retícula o urdimbre general. En pocas palabras, me adscribo a la estrategia de permitir que sean los datos los que guien nuestras conceptualizaciones y no a la inversa» (2).

De cuanto antecede cabe afirmar que la Psicología Diferencial, a través de estructuraciones como la aquí presentada, muestra con amplitud que la llamada «validez ecológica» nunca ha estado al margen de sus objetivos y presupuestos y que, en buena medida, la conforma. Es más, por haber partido de instrumentos progresivamente planificados para la resolución de los problemas sociales que recababan la colaboración del psicólogo-profesional, arriba ahora a la exposición de modelos de acción que sirven tanto para la investigación como para el ejercicio. Ši buena parte de las dimensiones identificadas factorialmente provienen y se basan en datos recopilados en torno al comportamiento humano entendido como producto, su recuperación a través de los constructos teóricos permite una homologación operativa en términos de procesos, útiles sobre todo el Psicología Aplicada así como en la investigación funcional y comprensiva de los mismos. La Psicología Diferencial, pues, sintoniza con los nuevos paradigmas en psicología fiel a sus métodos y herramientas, pero abierta una vez más a los desafíos tanto teóricos como, sobre todo, empíricos que la sociedad le ha demandado desde sus orígenes.

(1) Cattell, R. B. Personality and Learning Theory, volume 1: The structure of personality in its environment, New York, Springer, 1979, p. 205.

(2) Royce, J. R.: The conceptual framework for a multi-factor theory of individuality, en J. R. Royce (ed.): Multivariate Analysis and Psychological Theory, London, Academic Press, 1973 P. 391.

\section{Referencias}

VON BERTALANFFY, L.: Teoría General de Sistemas, México, Fondo de Cultura Económica, 1974.

CAPARROS, A.:Los paradigmas en psicología, Barcelona, Horsori, 1980.

CARROLL, J. S. y PAYNE, J. W.: Cognition and social behavior, New York, Lawrence Erlbaum Assoc. 1976.

CATTELL, R. B.: Handbook of multivariate experimental psychology, Chicago, Rand McNally, 1966.

-: Abilities: their structure, growth and action, Boston, Houghton-Mifflin, 1971.

-: Personality and Learning Theory, volume 1 : The structure of personality in its environment; volume 2: A systems theory of maturation and structured learning. New York, Springer, 1979 y 1980.

GUTTMAN, L.: A generalized simplex for factor analysis, en Psychometrika, 1955, 20, 173-192.

KUHN, T. S.: La estructura de las revoluciones cientificas, México, Fondo de Cultura EconómiCa, 1970.

LINDSAY, P. H. y NORMAN, D. A.: Procesamiento de la informaciön bumana: una introducción a la psicología, Madrid, Tecnos, 1975.

PAWLIK, K.:Right answers to the wrong questions? A re-examination of factor analytic personality research and its contribution to personality theory, en J. R. Royce: Multivariate Analysis and psychological theory, London, Academic Press, 1973. 


\section{Estudios}

PRIETO ZAMORA, J. M.: La personalidad ¿paradigma o sistema? en Estudios de Psicología, 1980,1 n. $^{\circ} 3$.

ROYCE, J. R.: Factors as theoretical constructs en American Psychologist, 1963, 18, 522-528.

-: Multivariate Analysis and Psychological Theory, London, Academic Press, 1973.

SCANDURA, J. M. y BRAINERD, C. J.: Structural/Process theories of complex buman behavior, Leiden, Sijthoff Int. Publ. Co., 1978. 